\title{
Necesidades tecnológicas en las instituciones públicas de educación superior de la Costa Oriental del Lago
}

\author{
Technological needs in public institutions of higher education on the East
} Coast of Lake

\section{Ireivi García}

ireivigarcia_06@hotmail.com

Código ORCID: 0000-0002-1966-4689

Universidad del Zulia. Maracaibo, Venezuela
- Articulo recibido en julio 2019

< Arbitrado en agosto 2019

< Publicado en enero 2020

\section{Resumen}

El estudio analizo las necesidades tecnológicas presentes en las instituciones públicas de educación superior en la Costa Oriental del Lago. Metodológicamente se tipificó como descriptiva, con diseño no experimental, transeccional y de campo. La población quedó constituida por las tres instituciones universitarias públicas ubicadas en la Costa Oriental del Lago. La técnica de recolección de datos fue la encuesta y el instrumento un cuestionario compuesto por 11 ítems, se utilizó el juicio de cinco expertos para la validez del mismo y el método de Alfa de Cronbach para su confiabilidad la cual arrojo un valor de 0,91. El análisis de los datos se calculó mediante la estadística descriptiva, a través de la media aritmética. Se evidencio que dentro de las necesidades tecnológicas que enfrentan las instituciones analizadas la capacidad técnica, plataforma tecnológica, los procesos, el control y la seguridad muestran una alta presencia.

Palabras clave: Capacidad técnica, control, necesidades tecnológicas, plataforma tecnológica, procesos, seguridad

\begin{abstract}
The study analyzed the technological needs present in public institutions of higher education on the East Coast of the Lake. Methodologically it was typified as descriptive, with a non-experimental, transectional and field design. The population was made up of the three public university institutions located on the East Coast of the Lake. The data collection technique was the survey and the instrument a questionnaire composed of 11 items, the judgment of five experts was used for its validity and the Cronbach's Alpha method for its reliability, which yielded a value of 0.91 . The analysis of the data was calculated using descriptive statistics, using the arithmetic mean. It was evidenced that within the technological needs that the analyzed institutions face, the technical capacity, technological platform, processes, control and security show a high presence.
\end{abstract}

Keywords: Technical capacity, control, technological needs, technological platform, processes, security 


\section{INTRODUCCIÓN}

En la actualidad, todas las empresas a nivel mundial deben adaptarse a las tecnologías de información y comunicación (TIC) y requieren estar lo más actualizadas posible para obtener el mayor nivel de competitividad y desarrollo, debido a que estas ayudan a que se ahorre tiempo, mejore la calidad, otorgan mayor status a las empresas, adecuan controles eficientes en su operación y por consiguiente le permite dar un paso firme para su progreso.

Por lo tanto, para toda organización es indispensable contar con una plataforma tecnológica, principalmente la de usuario final. Debido a que ésta comprende una serie de dispositivos que son la herramienta principal de los empleados, de lo contrario se interrumpiría su labor afectando las actividades del mismo y por ende, al proceso productivo del negocio.

Según Ávalos (2008) las diferentes organizaciones en Latinoamérica requieren asegurar no sólo que la tecnología con la que cuentan se utilice de forma adecuada, sino de la constante evaluación y adquisición de nuevas tecnologías promuevan la competitividad. Esto puede lograrse a través del empleo de la memoria tecnológica, debido a que es el registro sistemático de la información en sí, sea en las personas, equipos, organizaciones o procesos, cuyo objetivo es el de fomentar el aprendizaje tecnológico dentro de una organización.

Desde este contexto de aprendizaje, las universidades, en las últimas décadas, se han visto confrontadas con la necesidad de tener que redefinir su lugar dentro de un marco de cambio tecnológico acelerado $y$, en virtud del auge de los procesos de innovación, han adquirido un papel destacado como productoras de conocimiento valioso que puede ser aplicado en la solución de problemas, lo que, indudablemente, representa un aporte a la economía y la vida social de cualquier país o región.

En el caso específico de las instituciones públicas de educación superior de la Costa Oriental del Lago, se ha visto la necesidad de instaurar un proceso de gestión direccionado a la tecnología, donde se identifiquen sus necesidades, evidencie el progreso y desarrollo en sus diferentes etapas como: adquisición, adaptación, avance y obsolescencia, para lograr un mayor alcance y posicionamiento en cuanto a competitividad, calidad e innovación en el ámbito educativo.

Bajo esta óptica, se presenta este artículo donde se analizó las necesidades tecnológicas presentes en las instituciones públicas de educación superior en la Costa Oriental del Lago, de manera que sea utilizada como un instrumento que les permita desarrollarse en el ámbito tecnológico.

\section{Necesidades tecnológicas}

Una necesidad tecnológica es la expresión escrita, precisa y publica que una organización realiza de un requerimiento tecnológico acerca del cual la organización necesita implementar una solución (Matarranz, 2006). Refiere el autor que la necesidad de innovación tecnológica y la adaptación de las empresas a los cambios que se producen en su entorno son cuestiones de indudable interés y actualidad en el ámbito de la administración de empresas.

En el mismo orden de ideas, Cornella (1999) enfatiza que la actitud de las organizaciones ante la tecnología y su generación, selección, adquisición y utilización en aquellas tareas que conocen con la 
denominación genérica de actividades de investigación más desarrollado, es lo que permite a las empresas interpretar adecuadamente el proceso de mutación al que deben someterse.

Asimismo, Ávalos (2008) presenta la necesidad de adquisición de tecnología como un papel importante en la acumulación de capacidades referidas a ésta, por ello se le asigna el propósito general de convertir los métodos de compra de tecnología en procesos que permitan una verdadera transferencia de este recurso; orientando la gestión para adquirir las capacidades tecnológicas, y así usarla adecuadamente, adaptarla y mejorarla, más que a adquirir una capacidad productiva.

De acuerdo a lo anteriormente expuesto por los diferentes autores citados, la necesidad de adquirir tecnología así como el uso de la misma solo puede darse con el dominio de habilidades y destrezas que constituyen las capacidades tecnológicas, facilitando todas las actividades involucradas en el desarrollo tecnológico. Estas actividades forman parte de los recursos tecnológicos, incorporando un conjunto de medios materiales (maquinarias, equipos, entre otros), además de distintos elementos inmateriales como la propiedad intelectual, las bases de datos y de conocimientos.

\section{Capacidad técnica}

De acuerdo a las aseveraciones de Torres (2006), la capacidad técnica se encuentra íntimamente ligada a la habilidad existente en las organizaciones en función de mantener un nivel de productividad constante mediante el cual dicha organización logre la satisfacción de sus clientes, a través de la elaboración de productos de calidad y costos razonables, todo ello manteniendo una vigilancia constante de las potenciales nuevas exigencias del mercado con el objeto de realizar las adecuaciones que dichas exigencias demanden a razón de mantener su nivel de competitividad en el mercado.

Por su parte Avalos (2008), señala que las capacidades tecnológicas se manifiestan dentro de las empresas mediante los diferentes conocimientos adquiridos y acumulados de los propios procesos productivos, a estos conocimientos se le suele llamar el Know How, de igual manera posee un papel fundamental la capacidad de aplicar dichos conocimientos.

De acuerdo al autor citado anteriormente, este menciona que los gerentes poseen un papel preponderante en las organizaciones en su papel de evitar altos niveles de dependencia de entes externos, velando por realizar procesos de selección y asimilación óptimos de la tecnología adquirida, todo ello de la mano de un proceso de desarrollo de tecnologías propias.

Asimismo, Romero (2006) refiere que la capacidad técnica es el conjunto de conocimientos y habilidades que poseen los miembros de la empresa para realizar o ejecutar algo. Resalta el hecho de que una gran parte de ella se presta a corto plazo, dando la impresión de que se trata de servicios puntuales, probablemente suministrado al momento de la compra y en el momento en que la institución confronta un problema.

Según García y Navas (2005), la capacidad tecnológica es definida como la facultad genérica intensiva en conocimiento para movilizar conjuntamente distintos recursos científicos y técnicos, que permite el desarrollo de productos o procesos productivos innovadores, al servicio de la estrategia corporativa creadora de valor en condiciones de mercado. 
De acuerdo con Casanueve (2001), el nivel de dominio tecnológico alcanzado por la empresa indica el grado en que los conocimientos tecnológicos transferidos 0 desarrollados endógenamente fueron absorbidos y transformados en una capacidad tecnológica. La cual a su vez incluye los conocimientos -científicos y tecnológicosacumulados y la habilidad de hacer, comprender, utilizar y desarrollar estos conocimientos para producir nuevas tecnologías.

En este sentido, para la autora de esta investigación la capacidad técnica está orientada al potencial empresarial de una organización en función a su crecimiento constante en el ámbito técnico, ergonómico, ingenieril, productivo, y de seguridad higiene y ambiente, con un enfoque global capaz de aplicar paradigmas más amplios y con la flexibilidad necesaria para adaptarse a los cambios constantes del entorno globalizado y con miras hacia la excelencia de los procesos que permitan permanecer a la empresa en el tope de la cadena de producción satisfaciendo a los clientes.

En el aspecto universitario la implementación de la cultura tecnológica va de la mano con la profesionalización y actualización de toda la comunidad que hace vida en el recinto académico, alcanzando de esta manera los conocimientos, habilidades y destrezas necesarias para brindar a los estudiantes toda la información, atención, asesoría técnica y experticia como complemento de su preparación en aras de su profesionalización e inmersión en el mundo laboral

\section{Plataforma tecnológica}

Según Mendoza (2005), la plataforma tecnológica son una agrupación de entidades interesadas en un sector concreto, lideradas por la industria, con el objetivo de definir una agenda estratégica de investigación sobre temas estratégicamente importantes y con una gran relevancia social, en los cuales lograr los objetivos de crecimiento, competitividad y sostenibilidad dependen de los avances tecnológicos y de investigación a mediano y largo plazo.

Siguiendo con las aseveraciones del autor anteriormente citado las plataformas tecnológicas abordan problemas estratégicos en aquellos casos en que lograr el crecimiento, la competitividad y la sostenibilidad en futuros de avances tecnológicas decisivos. En aquellas se dan cita todas las partes interesadas, dirigidas por la industria, para definir los objetivos de investigación, desarrollo tecnológico a medio y largo plazo, así como para establecer una serie de directrices para su consecución. Lograr estos objetivos mejorara de manera significativa la vida cotidiana de los empresarios en numerosas áreas. Las plataformas tecnológicas desempeñan una función primordial para alinear mejor las prioridades de investigación con las necesidades de la industria.

De acuerdo a Cedeño (2014), en el ámbito universitario las plataformas tecnológicas definen las estrategias de investigación y desarrollo tecnológicos adecuados, brindando estrategias innovadoras en cada una de las áreas en que se aplican. Generalmente son promovidas por las universidades del sector privado, pero cuentan 
con la participación de los agentes científicos y tecnológicos para planificar de una forma integrada las agendas estratégicas de investigación, y el desarrollo para el corto, mediano y largo plazo.

La autora de la presente investigación enfatiza sobre las plataformas tecnológicas, en lo referentes académicos e institucionales sobre el efecto de las Universidades como instituciones que realizan la mayor parte de las actividades de investigación que se efectúan en los países desarrollados o en franco desarrollo. Las funciones y actividades de I\&D de estos actores tienen objetivos diversos como lo son el desarrollo de la capacidad científica y tecnológica institucional y nacional, pretendiendo lograr una capacidad científica y tecnológica mínima y generalizada y obtener una capacidad científica y tecnológica especializada en determinadas áreas.

De esta manera la aplicación de conocimientos científicos y tecnológicos a problemas socioeconómicos concretos hará una tendencia a transferir conocimientos científicos, tecnológicos y directivos al sector productivo, considerando en una perspectiva amplia que incluye a todos los demandantes de servicios universitarios.

\section{Procesos}

Mielke (2002) señala que un proceso está conformado por una cadena productiva integrada por un conjunto de operaciones necesarias para llevar a cabo la producción de un bien o servicio, que ocurren de forma planificada, y producen un cambio o transformación de materiales, objetos 0 sistemas.
De acuerdo a Castellanos (2007) los procesos relacionados con una adecuada gestión de la tecnología requieren la integración en un paquete tecnológico, entendido como el conjunto de conocimientos empíricos o científicos, nuevos o copiados, de acceso libre o restringido, jurídicos, comerciales o técnicos, necesarios para producir un bien o servicio.

Refiere el citado autor que en el proceso se ve reflejada la forma mediante la cual la organización productiva conoce, negocia, maneja y evalúa tecnologías, Involucrando también la búsqueda y selección de tecnologías, negociación y transferencia de tecnologías, monitoreo y prospectiva tecnológica, propiedad industrial, contratos de tecnología, asistencia técnica y servicios técnicos.

La investigadora hace un análisis del proceso, como elemento base de la gestión de tecnologías, y lo define como una secuencia de acciones $o$ pasos que deben seguirse 0 cumplirse a cabalidad y en los lapsos temporales justos para obtener de esta forma un producto final tangible o no, pero que a su vez sea capaz de generar un beneficio a la organización y a su entorno como un elemento más de la sustentabilidad y sostenibilidad organizacional.

A nivel universitario y ya dentro del ámbito académico el proceso de la gestión tecnológica debe ir de la mano con los lineamientos establecidos por la cultura educativa que se desea implantar e implementar, de modo que la aplicación de la ciencia, la producción y los fenómenos culturales generen bienestar a toda la familia del conocimiento y a su vez dejar sembrada la semilla que dará frutos a las generaciones futuras de universitarios. 


\section{Control}

Chiavenato (2009) expresa que el control es una función administrativa que mide, evalúa el desempeño y toma la acción correctiva cuando se necesita. De este modo, el control es un proceso esencialmente regulador por cuanto se hace parte del proceso administrativo junto con la planeación, organización, dirección y lo que la precede, como medio de regulación utilizado por un individuo o empresa, determinando los mecanismos para controlar y avalar su desempeño orientándose así a las decisiones. En tal sentido la empresa crea mecanismos de control e incentivos de los gestores con autonomía directiva que aminora las pérdidas por comportamientos inconscientes con sus objetivos.

En el mismo orden de ideas para el autor citado, el propósito de la función de control es tomar acciones correctivas necesarias para asegurar el cumplimiento de los objetivos de la institución considerando la misma como un sistema, donde sus diversas áreas funcionales establecen lazos de retroalimentación con el ambiente externo, dando lugar a un proceso auto-correctivo, que a su vez se complementa con el continuo intercambio de información que entre las mismas áreas debe ocurrir.

Para Castellanos (2007) el control sobre la aplicación de la tecnología tiene dos dimensiones: amplitud y profundidad, y puede hallarse más allá del campo de interés de una empresa. La amplitud del control, que es la dimensión más fácil de alcanzar, requiere que la empresa obtenga capacidad tecnológica en todas las etapas conducentes a la fabricación de un acuerdo de licencia. La profundidad del control se alcanza plenamente al final de un proceso de tres etapas, a saber: acceso, asimilación y supervisión.
De acuerdo a las aseveraciones del citado autor, para lograr el control, el sistema debe mantenerse informado acerca del conjunto de variables que son estratégicas y relevantes en su quehacer. Por tanto, es necesaria la presencia de mecanismos para la captura de información con calidad y oportunidad sobre las operaciones de dicho sistema y su impacto en el entorno.

Basado en la anteriormente expuesto, la investigadora se refiere al control, como el conjunto de pautas manifiestas que rigen cualquier proceso $\circ$ procedimiento, estableciendo las pautas para garantizar la calidad del producto o servicio, obteniendo así los estándares requeridos en función de las necesidades reales de la empresa.

Haciendo una transportación del control, dentro del sistema universitario como un todo, es necesario recalcar la labor de la supervisión metódica y estricta, de la observación asertiva y sobre todo de la aplicación de mecanismos correctivos (on the fly) al vuelo, que permitan enderezar las desviaciones durante la ejecución de alguna actividad, haciendo énfasis a los preceptos del aseguramiento de la calidad durante etapas específicas de la evolución del proceso.

\section{Seguridad}

De acuerdo a García (2000) es el conjunto de medidas técnicas, educacionales, médicas y psicológicas, empleadas para prevenir accidentes, tendientes a eliminar las condiciones inseguras del ambiente y a instruir - convencer a las personas acerca de la necesidad de la implementación de prácticas preventivas. Según el esquema organizacional de cada institución, los servicios de seguridad tienen el objetivo de establecer normas y procedimientos, gestionando los recursos 
posibles para conseguir la prevención de accidentes controlando los resultados obtenidos.

Así mismo Alexander (2007) dice que la seguridad en el proceso tecnológico debe gestionarse eficientemente creando procedimientos de seguridad tecnológica, los cuales se implantan y realizan un seguimiento continuo de la efectividad de dichos procedimientos. De allí se genera en concepto de sistema de gestión de seguridad de la información (SGSI), es una herramienta que sirve para proporcionar mecanismos de protección, y salvaguardar la información de los sistemas que la tratan o procesan de acuerdo con una serie de normas, políticas y procedimientos definidos por la institución.

La información anterior es conducente a la elaboración de conclusiones por parte de la investigadora, tomando en cuenta los preceptos básicos de la seguridad como un elemento preponderante en la salvaguarda de algún activo tangible o intangible y que a su vez el mismo surte efecto en la organización en la medida que sus aportes sean positivos y beneficiosos.

Así bien, la seguridad debe implementarse en el ambiente tecnológico universitario, de manera tal que la información, los datos, los procedimientos, las estructuras y los equipos permanezcan a salvo, y más aún permanezcan operativos de modo que sean capaces de mantenerse produciendo beneficios que apalanquen la gestión del conocimiento a través de la gestión tecnológica de la organización, cerrando así la brecha disfuncional que separa el éxito y los logros del atraso y la obsolescencia.

\section{MÉTODO}

Metodológicamente la investigación se tipificó como descriptiva, con diseño no experimental, transeccional y de campo. La población quedó constituida por las instituciones universitarias públicas ubicadas en la Costa Oriental del Lago, específicamente: La Universidad del Zulia Núcleo Costa Oriental del Lago, Universidad Nacional Experimental "Rafael María Baralt y el Instituto Universitario de Tecnología de Cabimas, cuya unidad informante estuvo compuesta por los coordinadores de programa y coordinadores por área, para un total de veinte sujetos.

La técnica de recolección de datos utilizada fue la encuesta y el instrumento un cuestionario con escala Lickert de cinco opciones de respuestas que van desde: totalmente de acuerdo (5), de acuerdo (4), indiferente (3), desacuerdo (2) y totalmente desacuerdo (1), compuesto por 11 ítems, se utilizó el juicio de 5 expertos para la validez del mismo y el método de Alfa de Cronbach para su confiabilidad el cual arrojo un valor de 0,91 , indicando que el instrumento posee muy alta confiabilidad. El análisis de los datos se calculó mediante la estadística descriptiva, a través de la media aritmética. Para tal fin se diseñó un baremo mostrado en la Tabla 1. 
Tabla 1. Baremo para la interpretación de la media aritmética

\begin{tabular}{cccc}
\hline ALTERNATIVA & RANGO & INTERVALO & CATEGORÍA \\
\hline Totalmente de acuerdo & 5 & $4,21-5,00$ & Muy alta presencia \\
De acuerdo & 4 & $3,43-4,20$ & Alta presencia \\
Indiferente & 3 & $2,62-3,42$ & Moderada presencia \\
Desacuerdo & 2 & $1,81-2,61$ & Baja presencia \\
Totalmente desacuerdo & 1 & $1,00-1,80$ & Muy baja presencia \\
\hline
\end{tabular}

Fuente: Elaboración propia

RESULTADOS

Como se evidencia en la Tabla 2, se muestra una media para la dimensión de 3,79 indicando que dentro de las necesidades tecnológicas actuales que enfrentan las instituciones públicas de educación superior en la Costa Oriental del Lago, la capacidad técnica, plataforma tecnológica, los procesos, el control y la seguridad muestran una alta presencia. En específico, todos los indicadores ostentan alta presencia, de la identificación de las necesidades tecnológicas tomando en cuenta cuales son las necesidades inmediatas con expectativas a medio o largo plazo.

Tabla 2. Dimensión: Necesidades tecnológicas

\begin{tabular}{lccl}
\hline DIMENSIÓN & INDICADOR & MEDIA & CATEGORÍA \\
\hline & Capacidad Técnica & 3,53 & Alta presencia \\
& Plataforma Tecnológica & 4,02 & Alta presencia \\
Necesidades & Procesos & 3,78 & Alta presencia \\
Tecnológicas & Control & 3,55 & Alta presencia \\
& Seguridad & 4,08 & Alta presencia \\
& PROMEDIO & $\mathbf{3 , 7 9}$ & Alta presencia \\
\hline
\end{tabular}

Fuente: Elaboración propia

Este alta presencia de la dimensión necesidades tecnológicas, valida lo expuesto por Ávalos (2008), para quien presenta la necesidad de adquisición de tecnología con un papel importante en la acumulación de capacidades referidas a ésta, por ello se le asigna el propósito general de convertir los métodos de compra de tecnología en procesos que permitan una verdadera transferencia de este recurso; orientando la gestión para adquirir las capacidades tecnológicas, y así usarla adecuadamente, adaptarla y mejorarla, más que a adquirir una capacidad productiva.

De igual manera validan la posición de la investigadora cuando afirma que, la necesidad de adquirir tecnología así como el uso de la misma solo puede darse con el 
dominio de habilidades y destrezas que constituyen las capacidades tecnológicas, facilitando todas las actividades involucradas en el desarrollo tecnológico.

Ahora bien, al detalle de cada uno de los indicadores utilizados para medir la dimensión necesidades tecnológicas (capacidad técnica, plataforma tecnológica, los procesos, el control y seguridad), a continuación se especifican los resultados obtenidos al respecto, mostrados en la tabla 2 , donde se recoge el resultado de la dimensión y cada uno de sus indicadores.

De esta forma, se evidencia para el indicador capacidad técnica, un promedio de 3,53 indicando alta presencia de los recursos estratégicos con los que cuentan las instituciones bajo estudio, para ello el personal que trabaja en la aplicación de las nuevas tecnologías es seleccionado por medio de pruebas de conocimiento en el área técnica. De esta manera, los resultados obtenidos coinciden con las afirmaciones de Romero (2006), quien asevera que la capacidad técnica es el conjunto de conocimientos y habilidades que poseen los miembros de la empresa para realizar o ejecutar algo.

Por otra parte, dichos resultados validan lo expuesto por la investigadora, al considerar que la capacidad técnica va de la mano con la profesionalización y actualización de toda la comunidad que hace vida en el recinto académico, alcanzando de esta manera los conocimientos, habilidades y destrezas necesarias para brindar a los estudiantes toda la información, atención, asesoría técnica y experticia como complemento de su preparación en aras de su profesionalización e inmersión en el mundo laboral.

En lo que concierne al indicador plataforma tecnológica, se observa una media de 4,02, indicando alta presencia de este indicador en las necesidades tecnológicas.

Tabla 3. Dimensión: Necesidades tecnológicas

\begin{tabular}{|c|c|c|c|}
\hline \multicolumn{4}{|c|}{ INDICADOR: CAPACIDAD TÉCNICA } \\
\hline $\mathrm{N}^{\circ}$ & ÍTEMS & $\mathrm{x}$ & CATEGORÍA \\
\hline 1 & La tecnología es uno de los recursos estratégicos con los que cuenta. & 3,60 & Alta presencia \\
\hline 2 & $\begin{array}{l}\text { El personal que trabaja en la aplicación de las nuevas tecnologías se } \\
\text { selecciona por medio de pruebas de conocimiento en el área técnica. }\end{array}$ & 3,45 & Alta presencia \\
\hline & Promedio Indicador & 3,53 & Alta presencia \\
\hline \multicolumn{4}{|c|}{ INDICADOR: PLATAFORMA TECNOLÓGICA } \\
\hline $\mathrm{N}^{\circ}$ & ÍTEMS & $\mathrm{x}$ & CATEGORÍA \\
\hline 3 & $\begin{array}{l}\text { Están vinculadas las prioridades de investigación con las } \\
\text { necesidades del sector industrial. }\end{array}$ & 4,05 & Alta presencia \\
\hline 4 & $\begin{array}{l}\text { La investigación y el desarrollo son elementos primordiales que } \\
\text { establecidos en las necesidades tecnológicas }\end{array}$ & 4,20 & Alta presencia \\
\hline \multirow[t]{2}{*}{5} & $\begin{array}{l}\text { Dentro del proceso tecnológico se cumplen una serie de etapas } \\
\text { sistemáticas para alcanzar el éxito del mismo }\end{array}$ & 3,80 & Alta presencia \\
\hline & Promedio Indicador & 4,02 & Alta presencia \\
\hline
\end{tabular}

Fuente: Elaboración propia (2019) 


\section{INDICADOR: CAPACIDAD TÉCNICA}

6 Los procesos tecnológicos realizados parten de la planificación.

3,95 Alta presencia

Las actividades realizadas en los departamentos están estrechamente relacionadas con el proceso tecnológico

3,60 Alta presencia

Promedio Indicador $3,53 \quad$ Alta presencia

INDICADOR: CONTROL

$\mathrm{N}^{\circ}$

8 Se controla el proceso

9

El desempeño de la gestión es evaluada por medio de la utilización de indicadores

ÍTEMS

\section{$x$}

3,45 Alta presencia

3,65 Alta presencia

Promedio Indicador

INDICADOR: SEGURIDAD

$\mathrm{N}^{\circ}$ ÍTEMS

10 Se utiliza como herramienta el sistema de gestión de seguridad de la información (SGSI) como mecanismo de protección.

11 Existen procedimientos definidos, normas y políticas para salvaguardar la información de los sistemas que la tratan o procesan.
3,55 Alta presencia

$X \quad$ CATEGORÍA

4,05 Alta presencia

$4,10 \quad$ Alta presencia

Promedio Indicador $4,08 \quad$ Alta presencia

PROMEDIO DIMENSIÓN $\quad 3,79 \quad$ Alta presencia

Esta situación permite afirmar que, se da alta presencia a las siguientes actividades: están vinculadas las prioridades de investigación con las necesidades del sector industrial (4.05); la investigación y el desarrollo son elementos primordiales que establecidos en las necesidades tecnológicas $(4,20)$ y dentro del proceso tecnológico se cumplen una serie de etapas sistemáticas para alcanzar el éxito del mismo $(3,80)$.

Con estos resultados se logra validar a Mendoza (2005), para quien la plataforma tecnológica es una agrupación de entidades interesadas en un sector concreto, lideradas por la industria, con el objetivo de definir una agenda estratégica de investigación sobre temas estratégicamente importantes y con una gran relevancia social, en los cuales lograr los objetivos de crecimiento, competitividad y sostenibilidad dependen de los avances tecnológicos y de investigación a mediano y largo plazo.

Así entonces, los resultados presentados validan la opinión de la investigadora para quien la plataforma tecnológica en el sector universitario se vincula con las funciones $y$ actividades de I\&D, dado que tienen objetivos diversos como lo son el desarrollo de la capacidad científica, tecnológica institucional y nacional.

En lo que concierne al indicador diseño del proceso, se observa una media de 3,53, la cual indica alta presencia de este indicador, al considerar los encuestados que: los procesos 
tecnológicos realizados parten de la planificación $(3,95)$ y las actividades realizadas en los departamentos están estrechamente relacionadas con el proceso tecnológico $(3,60)$.

Los resultados demuestran alta coincidencia con lo expuesto por Castellanos (2007), para quien los procesos relacionados con una adecuada gestión de la tecnología requieren la integración en un paquete tecnológico, entendido como el conjunto de conocimientos empíricos o científicos, nuevos o copiados, de acceso libre o restringido, jurídicos, comerciales o técnicos, necesarios para producir un bien o servicio.

Sumado a lo expuesto, se validan las afirmaciones realizadas por la investigadora sobre el proceso, al considerarlos como una secuencia de acciones o pasos, que deben seguirse o cumplirse a cabalidad y en los lapsos temporales justos para obtener de esta forma un producto final tangible o no, pero que a su vez sea capaz de generar un beneficio a la organización y a su entorno como un elemento más de la sustentabilidad y sostenibilidad organizacional.

En cuanto al indicador control, se aprecia una media de 3,55, otorgándole alta presencia al responder los encuestados que se controla el proceso tecnológico por medio del acceso, asimilación y supervisión y el desempeño de la gestión es evaluada por medio de la utilización de indicadores.

Sobre la base de los resultados, se evidencia alta concordancia con lo expuesto por Castellanos (2007), quien expresa que para lograr el control, el sistema debe mantenerse informado acerca del conjunto de variables que son estratégicas y relevantes en su quehacer. Por tanto, es necesaria la presencia de mecanismos para la captura de información con calidad y oportunidad sobre las operaciones de dicho sistema y su impacto en el entorno.

En virtud a esto, la autora valida los resultados mostrados, puesto que dentro del sistema universitario como un todo, el control es necesario para recalcar la labor de la supervisión metódica y estricta, donde la observación asertiva y la aplicación de mecanismos correctivos, permitan enderezar las desviaciones durante la ejecución de alguna actividad, haciendo énfasis a los preceptos del aseguramiento de la calidad durante etapas específicas de la evolución del proceso.

Prosiguiendo el análisis, se verifican los resultados obtenidos para el indicador seguridad. Se observa una media de 4,08, indicando alta presencia de este indicador dado que se utiliza como herramienta el sistema de gestión de seguridad de la información (SGSI) como mecanismo de protección y existen procedimientos definidos, normas y políticas para salvaguardar la información de los sistemas que la tratan o procesan.

Los resultados coinciden con Alexander (2007), quien asevera que la seguridad en el proceso tecnológico debe gestionarse eficientemente creando procedimientos de seguridad tecnológica, los cuales se implantan y realizan un seguimiento continuo de la efectividad de dichos procedimientos. Así bien, la investigadora valida los resultados referidos a la seguridad planteado que esta debe implementarse en el ambiente tecnológico universitario, de manera tal que la 
información, los datos, los procedimientos, las estructuras y los equipos permanezcan a salvo, y más aún permanezcan operativos de modo que sean capaces de mantenerse produciendo beneficios que apalanquen la gestión del conocimiento a través de la gestión tecnológica de la organización, cerrando así la brecha disfuncional que separa el éxito y los logros del atraso y la obsolescencia.

Vistos estos resultados, se verifica que en las instituciones bajo estudio valoran la necesidad de adquirir tecnología así como el uso de la misma y esta solo puede darse con el dominio de habilidades y destrezas que constituyen las capacidades tecnológicas, facilitando todas las actividades involucradas en el desarrollo tecnológico.

\section{CONCLUSIONES}

Las necesidades tecnológicas presentes en las instituciones públicas de educación superior en la Costa Oriental del Lago, como objetivo inicial de la investigación en curso, presento durante su estudio y análisis un resultado orientado hacia una alta presencia de cumplimiento y acción, repercutiendo esto directamente sobre la capacidad técnica, plataforma tecnológica, los procesos, el control y la seguridad, lo que se traduce en el fortalecimiento de todas las áreas conjuntas, en torno a la adquisición y generación de tecnologías emergentes, así como también su adquisición y utilización eficaz, efectiva y eficiente, hacia el logro de los objetivos de I+D, convirtiendo a las organizaciones educativas en fuentes inagotables de recursos materiales, humanos y técnicos, que apalancaran de seguro el crecimiento del país, cerrando de esta manera la brecha tecnológica creada por las erráticas políticas de inversión acción.

\section{REFERENCIAS}

Alexander, A. (2007). Diseño de un sistema de gestión de seguridad de la información. Bogotá. Colombia

Ávalos, I. (2008). Memoria tecnológica. Documento en Línea. Disponible en: http://www.loctiblog.com/como-hacer/lamemoria-tecnologica-de-la-empresa36.html

Casanueve, C. (2001). "The adquisition of firm technological capabilities in Mexico's open economy, the case of vitro". Technological forecastig and Social change 66, 1:75-85

Castellanos, O. (2007). Gestión tecnológica, de un enfoque tradicional a la inteligencia. Facultad de Ingeniería Universidad Nacional de Colombia Bogotá D.C. - Colombia

Cedeño, P. (2014), Gestión tecnológica en instituciones privadas de educación superior de la Costa Oriental del Lago. Postgrado en Gerencia, Mención Operaciones. Universidad del Zulia. Cabimas-Venezuela

Chiavenato, I. (2009). Comportamiento organizacional. McGraw-Hill. Colombia

Cornella, A. (1999). Los recursos de la información. Ventaja competitiva de las empresas. Editorial McGraw-Hill-Madrid, España

García, A. (2000). Planeación estratégica y planeación tecnológica. En Conceptos Generales de Gestión Tecnológica Colección Ciencia y Tecnología No. 27. BID - SECAB - CINDA

García, F. y Navas, J. (2005). "Estudio empírico del sostenimiento de las ventajas competitivas de bases tecnológica. Un análisis del sector biotecnológico español". En XI Seminario LatinoIberoamericano de gestión tecnológica. Salvador de Bahia, Brasil 
Matarranz, A. (2006). Ideas y actualidad sobre Marketing, tecnología a innovación. Documento electrónico, disponible en la página web: www.innovationmarketingmexico.eordpre ss.com

Mendoza, A. (2005). Manual para determinar necesidades de capacitación. Editorial Trillas México

Mielke, E. (2002). Análisis de la cadena productiva de comercialización. Edición Conceito cadeia produtiva 35. Madeira, Portugal
Romero, R. (2006). Marketing. Editorial Palmir E.I.R. Madrid, España

Torres, A. (2006). Aprendizaje y construcción de capacidades tecnológicas. Journal of Technology Management \& Innovation, 1 (5), pp. 12-24

Villamizar, R. y Mondragón, J. (1996). Lecciones de los países del Asia-Pacífico en tecnología, productividad y competitividad. Editorial Norma. Santa Fe de Bogotá 